\title{
HPP1: A transmembrane protein-encoding gene commonly methylated in colorectal polyps and cancers
}

\author{
Joanne Young*, Kelli G. Biden*†, Lisa A. Simms*, Phillip Huggard, Rozemary Karamatic*, Helen J. Eyre§, \\ Grant R. Sutherland ${ }^{\S}$, Nirmitha Herath ${ }^{\ddagger}$, Melissa Barker", Gregory J. Anderson ${ }^{\ddagger}$, David R. Fitzpatrick ${ }^{\ddagger}$, \\ Grant A. Ramm ${ }^{\ddagger}$, Jeremy R. Jass", and Barbara A. Leggett*
}

*Conjoint Gastroenterology Laboratory, Royal Brisbane Hospital Foundation Clinical Research Centre, Bancroft Centre, Herston 4029, Australia; ${ }^{\circledR}$ Centre for Medical Genetics, Women's and Children's Hospital, Adelaide 5006, Australia; ${ }^{\ddagger}$ Queensland Institute of Medical Research, 300 Herston Road, Herston 4029, Australia; and "Department of Pathology, University of Queensland, Herston 4006, Australia

Edited by Bert Vogelstein, Johns Hopkins Oncology Center, Baltimore, MD, and approved October 30, 2000 (received for review August 29, 2000)

\begin{abstract}
Adenomas are the precursors of most colorectal cancers. Hyperplastic polyps have been linked to the subset of colorectal cancers showing DNA microsatellite instability, but little is known of their underlying genetic etiology. Using a strategy that isolates differentially methylated sequences from hyperplastic polyps and normal mucosa, we identified a 370-bp sequence containing the $5^{\prime}$ untranslated region and the first exon of a gene that we have called HPP1. Rapid amplification of CDNA ends was used to isolate HPP1 from normal mucosa. Using reverse transcription-PCR, HPP1 was expressed in 28 of $\mathbf{3 0}(\mathbf{9 3 \%})$ normal colonic samples but in only seven of $30(23 \%)$ colorectal cancers $(P<0.001)$. The $5^{\prime}$ region of HPP1 included a CpG island containing 49 CpG sites, of which $96 \%$ were found to be methylated by bisulfite sequencing of DNA from colonic tumor samples. By COBRA analysis, methylation was detected in six of nine (66\%) adenomas, 17 of $27(63 \%)$ hyperplastic polyps, and 46 of $55(84 \%)$ colorectal cancers. There was an inverse relationship between methylation level and mRNA expression in cancers $(r=-0.67 ; P<0.001)$, and 5-aza-2-deoxycytidine treatment restored HPP1 expression in two colorectal cancer cell lines. In situ hybridization of HPP1 indicated that expression occurs in epithelial and stromal elements in normal mucosa but is silenced in both cell types in early colonic neoplasia. HPP1 is predicted to encode a transmembrane protein containing follistatin and epidermal growth factor-like domains. Silencing of HPP1 by methylation may increase the probability of neoplastic transformation.
\end{abstract}

$\mathbf{T}$ he evolution of colorectal cancer occurs as a stepwise process described at the morphological level as the adenomacarcinoma sequence. Inherited colorectal cancer syndromes have yielded fundamental insights into the underlying molecular mechanisms. In familial adenomatous polyposis, inactivation of the "gatekeeper" APC gene initiates the development of multiple adenomas by disrupting growth control. Most sporadic colorectal neoplasms are believed to be initiated by a similar mechanism, and subsequent progression occurs through the development of chromosomal instability. In hereditary nonpolyposis colorectal cancer, adenoma progression is accelerated through the early inactivation of a "caretaker" DNA mismatch repair gene and establishment of DNA instability (1). A deficiency of DNA mismatch repair is also implicated in the subset of sporadic colorectal cancers showing DNA microsatellite instability (MSI); the usual underlying mechanism is silencing of the DNA mismatch repair gene $h M L H 1$ by promoter methylation (2). However, sporadic adenomas rarely show either MSI (3) or loss of expression of DNA mismatch repair genes except in the context of hereditary nonpolyposis colorectal cancer $(3,4)$. Moreover, the frequency of APC mutation is reduced in sporadic MSI cancers $(5,6)$. These observations cast doubt on the role of adenoma as the precursor of sporadic MSI-high colorectal cancer.

Hyperplastic polyps are less well characterized than adenomas but are related epidemiologically to colorectal cancers (7). In normal colorectal mucosa, epithelial cells mature as they migrate along the crypt column and are lost from the surface epithelium (8). In hyperplastic polyps, there is delayed cell migration, and hypermature cells are retained within the surface epithelium (9). These observations suggest a primary defect in migration or exfoliation, but the mechanism is unknown. The risk of colorectal cancer is increased in subjects developing multiple hyperplastic polyps (10, 11), and hyperplastic polyps may show clonal genetic alterations that are also described in colorectal cancer. These include K-ras mutations (12), allele loss on chromosome 1p (13), and transforming growth factor $\beta$ RII mutations (14). K-ras may be an initiating mutation in small hyperplastic polyps $(13,15)$. However, the mechanism initiating large multiple and proximally located hyperplastic polyps, which have been linked to cancers showing DNA MSI $(13,16,17)$, is unknown.

A methylator phenotype in which some promoter sequences are hypermethylated recently has been shown to be associated with a subset of colorectal cancers, notably cancers with MSI $(18,19)$. Because hyperplastic polyps may show MSI, methylation may have had a role in their initiation. Using a strategy that isolates differentially methylated sequences from colonic neoplasms and corresponding normal colonic mucosa, we identified a sequence of 370 bp containing the 5 -untranslated region and the first exon of a novel gene, which we have called HPP1, because it initially was identified in subjects with hyperplastic polyposis. This report describes the isolation and characterization of HPP1.

\section{Materials and Methods}

Samples. This study was carried out on a subset of 55 sporadic colorectal cancers from a series of 303 previously characterized according to their level of DNA MSI (20). Of these, 19 were high-level MSI, 18 were low-level MSI, and 18 were microsatellite stable. Also included were 10 adenomas and 27 hyperplastic polyps freshly collected from patients with hyperplastic polyposis. DNA was extracted from all samples by a salt precipitation technique (21).

Global Methylation Screening Assay. Paired normal and neoplastic DNA samples from individuals with hyperplastic polyposis and

This paper was submitted directly (Track II) to the PNAS office.

Abbreviations: MSI, microsatellite instability; UTR, untranslated region; RACE, rapid amplification of CDNA ends; RT-PCR, reverse transcription-PCR; AP, arbitrarily primed; 5-AzaC, 5-aza-2-deoxycytidine; ISH, in situ hybridization; SMA, smooth muscle actin; LOH, loss of heterozygosity.

†To whom reprint requests should be addressed. E-mail: kelliB@qimr.edu.au.

The publication costs of this article were defrayed in part by page charge payment. This article must therefore be hereby marked "advertisement" in accordance with 18 U.S.C. $\$ 1734$ solely to indicate this fact.

Article published online before print: Proc. Natl. Acad. Sci. USA, 10.1073/pnas. 011415298. Article and publication date are at www.pnas.org/cgi/doi/10.1073/pnas.011415298 
(a)

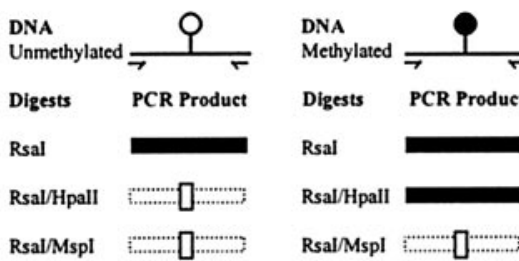

(b)

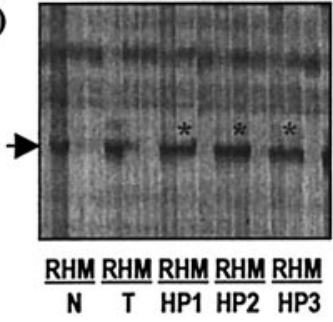

(c)

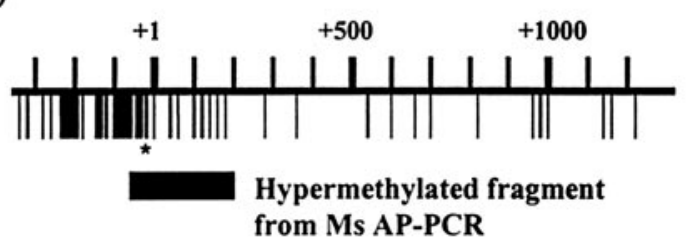

(d)

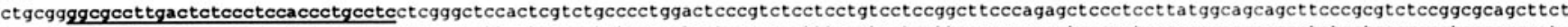

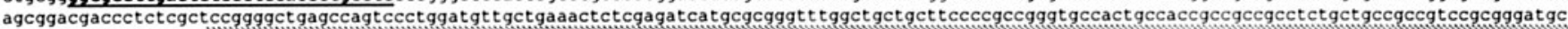

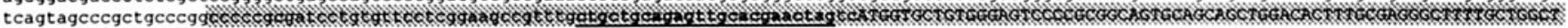
H

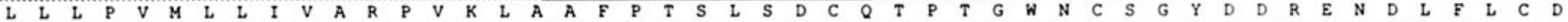
CACCAACACCTGTAAATTTGATGGGGAATGTTTAAGAATTGGAGACACTGTGACTTGCGTCTGTCAGTTCAAGTGCAACAATGACTATGTGCCTGTGTGTGGCTCCAATGGGGAGAGCTACCAGAATGAGTGTTACCTGCGACA

$\begin{array}{llllllllllllllllllllllllllllllllllllllllllllllllllllll}T & N & T & C & K & F & D & G & E & C & L & R & I & G & D & T & V & T & C & V & C & Q & F & K & C & N & N & D & Y & V & P & V & C & G & S & N & G & E & S & Y & Q & N & E & C & Y & L & R & Q\end{array}$ GGCTGCATGCAAACAGCAGAGTGAGATACTTGTGGTGTCAGAAGGATCATGTGCCACAGATGCAGGATCAGGATCTGGAGATGGAGTCCATGAAGGCTCTGGAGAAACTAGTCAAAAGGAGACATCCACCTGTGATATTTGCCA

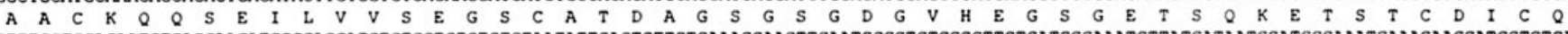
GTTTGGTGCAGAATGTGACGAAGATGCCGAGGATGTCTGGTGTGTGTGTAATATTGACTGTTCTCAAACCAACTTCAATCCCCTCTGCGCTTCTGATGGGAAATCTTATGATAATGCATGCCAAATCAAAGAAGCATCGTGTCA

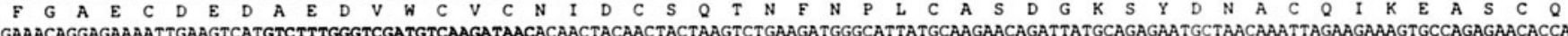
GAAACAGGAGAAAATTGAAGTCATGTCTTTGGGTCGATGTCAAGATAACACAACTACAACTACTAAGTCTGAAGATGGGCATTATGCAAGAACAGATTATGCAGAGAATGCTAACAAATTAGAAGAAG CATACCTTGTCCGGAACATTACAATGGCTTCTGCATGCATGGGAAGTGTGAGCATTCTATCAATATGCAGGAGCCATCTTGCAGGTGTGATGCTGGTTATACTGGACAACACTGTGAAAAAAAGGACTACAGTGTTCTATACGT

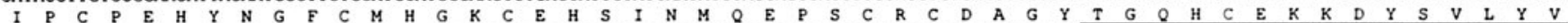
TGTTCCCGGTCCTGTACGATTTCAGTATGTCTTAATCGCAGCTGTGATTGGAACAATTCAGATTGCTGTCATCTGTGTGGTGGTCCTCTGCATCACAAGGGCCAAACTTTAGGTAATAGCATTGGACTGAGATTTGTAAACTT

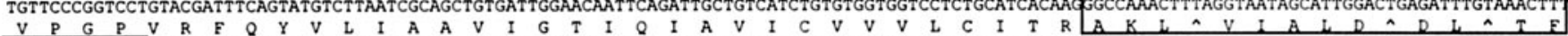
CCAACCTTCCAG AAATGCCCCAGAAGCAACAGAATTCACAGACAGAAGCAAAATACAGGGCACTACAGTTCAGACAATACAACAAGAGCGTCCACGAGGTTAATCTAAagggagCat gt $t$ t Cacagt ggCtggact accgaga

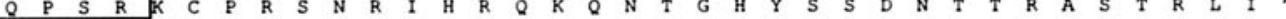

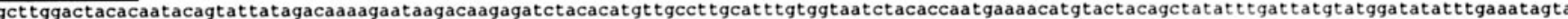
tacattgtettgatgettttctgtaatgtaaataaactatttatatcac

(e)

\begin{tabular}{|c|c|c|}
\hline MP2 (E14368) & 301 aa & 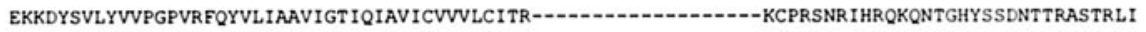 \\
\hline TENB2 (AF179274) & 301 aa & YVLIAAVIGTIQIAVICVVVLCITR--- \\
\hline TMEFF2 (AB017269) & 301 aa & 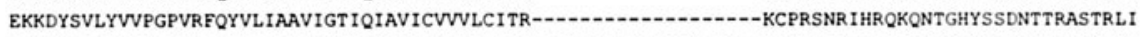 \\
\hline HSM802469 & 301 aa & GPVRFQYVLIAAVIGTIQIAVICVVVLCITRAKL^VIALD^DL^TFQPSRKCPRSNRIHRQKQNTGHYSSDNTTRASTRLI \\
\hline P1 & 301 aa & EKKDYSVLYVVPGPVRFQYVLIAAVIGTIQIAVICVVVLCITRAKL^VIALD^DL^TFQPSRKCPRSNRIHRQKQNTGHYSSDNTTRASTRLI \\
\hline moregulin & 301 aa & KKDYSVVLYVVPGPVRFQYVLI IAAVIGT IQIAVI ICVVVLCI TRR \\
\hline
\end{tabular}

Fig. 1. (a) Methylation-sensitive arbitrarily primed PCR (Ms AP-PCR) schematic representation (22). (b) Hypermethylated fragment (*) from Ms AP-PCR in colon tumor (T) and hyperplastic polyps (HP) of a single patient. N, normal colonic mucosa; R, Rsal digest; $\mathrm{H}$, Rsal/Hpall digest; M, Rsal/Mspl digest. (c) Distribution of CpG sites across HPP1 cDNA sequence. CpG sites are indicated by tick marks; the CpG site analyzed for methylation by COBRA is indicated by *. (d) Nucleotide sequence of HPP1 CDNA and predicted amino acid sequence. Shaded box indicates position of hypermethylated fragment. Primer sequences used for sequencing of overlapping fragments are underlined; forward primers are in bold type. Open box indicates 57-base insertion with stop codons indicated $\left({ }^{\wedge}\right)$. (e) Alignment of $3^{\prime}$-amino acid sequences for homologous CDNAs. Homologous sequence is indicated $(*)$, as are stop codons $\left({ }^{\wedge}\right)$.

cancer were digested with RsaI to create smaller fragments and further digested with restriction enzymes having differential sensitivities to cytosine methylation (MspI and HpaII), before undergoing arbitrarily primed PCR amplification (ref. 22; Fig. $1 a)$ in the presence of ${ }^{33} \mathrm{P}-\mathrm{dATP}$. Fragments were resolved on sequencing gels, and those showing differential methylation were eluted from the gel, cloned into pGEM-T vectors (Promega), and sequenced. Fragment sequences underwent BLAST searches against GenBank and European Molecular Biology Laboratory databases.

Confirmation of Methylation. Methylation of $\mathrm{CpG}$ sites within HPP1 was confirmed by using bisulfite sequencing (23) and nested primer sets (HPBisF 5'-GGCGTTTTGATTTTTTTTTTATTTTGTTTT-3' and HPBisR 5'-AACGCGCTAAAATAAACTAATCTATACTAA- $3^{\prime}$ as the outer set and HPBisF2 5'-GTTTTTTAGAGTTTTTTTTTTATGGTAGTAGT-3' and HPBisR2 5'-AACAATCACTTAAAAAAATAAAAAAAACAA- $3^{\prime}$ as the inner set) in nine putatively methylated and five unmethylated DNA samples analyzed by arbitrarily primed (AP)-PCR. To screen larger numbers of samples for HPP1specific methylation, we used a COBRA assay (24) to produce restriction fragments from bisulfite-treated DNA only if methylation was present. We used the primers described above to amplify fragments of $427 \mathrm{bp}$, which were cleaved into fragments of 271 and 156 bp by digestion with $R s a \mathrm{I}$. Cleavage fragments were quantified by densitometry. Results of digestion by $R s a \mathrm{I}$ correlated with the results of bisulfite sequencing of the $\mathrm{CpG}$ site that formed part of the RsaI recognition sequence in all 14 samples analyzed by AP-PCR, as well as $\mathrm{CpG}$ dinucleotides in the surrounding DNA. A control digest was performed with AluI, which digested DNA that had not undergone bisulfite modification, and this was used to check for incomplete conversion. HPP1 COBRA assays were performed on 55 sporadic colorectal cancers, 10 adenomatous polyps, and 27 hyperplastic polyps as well as corresponding normal mucosa samples.

Sequencing of Full-Length cDNA and Rapid Amplification of CDNA Ends (RACE). cDNA was synthesized from $3 \mu \mathrm{g}$ total RNA from pooled normal colonic mucosa using Superscript II (GIBCO/BRL) reverse transcriptase and random hexamers. A 506-bp cDNA fragment spanning a portion of the $5^{\prime}$ untranslated region (UTR) was amplified with a downstream primer within the methylated fragment (HPX 5'-GGCAGTCACTTAAGGAGGTAGGGAAAGCAG-3') and an upstream primer designed from an extended region of homologous database sequence (HPA 5'-GGCGCCTTGACTCTCCCTCCACCCTGCCTC-3'). Similarly, a 781-bp cDNA fragment was produced from an upstream primer within the methylated fragment (HPC 5'CTGCTGCAGAGTTGCACGAACTAG-3') and a downstream primer in the coding portion of homologous database sequence (HPV 5'-GTTAGCATTCTCTGCATAATCTGTTCTTGC- $\left.3^{\prime}\right)$. These overlapping fragments were sequenced and checked for homology to database entries. In addition, a further 
828-bp fragment was sequenced, which contained the cDNA equivalent portion of the methylated fragment (HPA and HPW 5'-TGACTAGTTTCTCCAGAGCCTTCAT-3'). Then, 3' RACE was performed with human colonic mucosa $3^{\prime}$-RACEReady cDNA and human colorectal cancer $3^{\prime}$-RACE-Ready cDNA (CLONTECH). The template for 3' RACE was derived from either five pooled normal mucosa total RNA samples or seven pooled tumor total RNA samples known to express HPP1 by reverse transcription-PCR (RT-PCR). Amplification was carried out with the sense primer NGSP2 (5'-GTTAGCATTCTCTGCATAATCTGTTCTTGC-3' designed to overlap with the 781-bp fragment described above), an antisense modified oligo(dT) primer, Advantage 2 polymerase (CLONTECH), and a touchdown PCR program. PCR products were excised from agarose gels and subcloned into pGEM-T vectors (Promega) for sequencing.

RT-PCR. RT-PCR of HPP1 was performed on a subset of cases in which fresh frozen tissue was available (30 cancers, 1 adenoma, and 1 hyperplastic polyp) and on which COBRA assays had already been performed. RNA was extracted from paired normal and tumor tissues using RNA Isolation Reagent (ABgene) according to the manufacturer's recommendations. cDNA was synthesized as described above. Primers were designed (HPC and HPZ 5' -TGAACTGACAGACGCAAGTCAC-3') that amplified a 306-bp fragment spanning a portion of the 5' UTR and coding sequence of $H P P 1$. cDNA products were resolved on $2 \%$ agarose gels and stained briefly in $0.5 \mu \mathrm{g} / \mathrm{ml}$ ethidium bromide. Loading was controlled by the simultaneous PCR of $\beta$-actin cDNA (25). RT-PCR primers spanned an intron of HPP1 and hence were unlikely to amplify genomic DNA.

Manipulation of HPP1 Expression by 5-Aza-2-Deoxycytidine (5-AzaC). HT29 adenocarcinoma (microsatellite stable) cells and Lovo (high-level MSI) cells (American Type Culture Collection) are two colorectal cancer lines that do not express HPP1 by RTPCR, and in which HPP1 DNA is $100 \%$ methylated by COBRA assay. The demethylating agent 5-AzaC (Sigma) was used to reverse the methylation-suppressed expression of HPP1 (26). HT29 and Lovo were seeded at $1 \times 10^{5} \mathrm{cells} / \mathrm{ml}$ in $10 \mathrm{ml}$ of RPMI and exposed to concentrations of 5-AzaC ranging from 0 to 10.0 $\mu \mathrm{M}$ after $48 \mathrm{~h}$ of growth. Cells were harvested after $72 \mathrm{~h}$ of exposure, and RNA was extracted. A final concentration of 0.5 $\mu \mathrm{M} 5$-AzaC produced optimal induction of HPP1 expression without cytotoxicity. Both lines were again seeded as described above to study the timing of HPP1 expression induction. Cells were grown in $0.5 \mu \mathrm{M}$ with 5 -AzaC for $72 \mathrm{~h}$ and then incubated without 5-AzaC for a further $72 \mathrm{~h}$. Cells were harvested immediately before and at 12-h intervals after the initial contact with 5-AzaC. All cells harvested underwent RT-PCR for HPP1.

In Situ Hybridization (ISH) of HPP1 mRNA. For detection of HPPI mRNA in paraffin-embedded tissue, an 828-bp fragment of HPPI cDNA was subcloned into a pGEM-T vector. A 300-bp fragment for ISH was produced by alkaline hydrolysis. Digoxigenin-labeled riboprobes, for sense (control) and antisense, were produced for HPPI by in vitro transcription with SP6 and T7 polymerases as described (27). ISH was performed on $5-\mu \mathrm{m}$ sections, deparaffinized by xylol, and rehydrated by gradient alcohol before exposure to hydrochloric acid ( $0.2 \mathrm{~mol} / \mathrm{liter})$, as described (28). Sections were permeabilized with $5 \mathrm{mg} / \mathrm{ml}$ proteinase $\mathrm{K}$ at $37^{\circ} \mathrm{C}$ for $15 \mathrm{~min}$, followed by fixation in $4 \%$ paraformaldehyde for $20 \mathrm{~min}$ at room temperature. Prehybridization $(50 \%$ formamide $/ 1 \% \mathrm{SDS} / 5 \times$ standard saline citrate/ $500 \mathrm{mg} / \mathrm{ml} \mathrm{tRNA} / 50 \mathrm{mg} / \mathrm{ml}$ heparin) was performed at $67.5^{\circ} \mathrm{C}$ for $4 \mathrm{~h}$ followed by hybridization for $16 \mathrm{~h}$ at $67.5^{\circ} \mathrm{C}$ in a solution containing $1 \mathrm{mg} / \mathrm{ml}$ digoxigenin-labeled riboprobe. Sections were washed of unbound probe and incubated with alkaline phosphatase-conjugated antidigoxigenin polyclonal sera (1:200) for $2 \mathrm{~h}$. Unbound antibody was removed by washes, followed by visualization with nitroblue tetrazolium chloride/5-bromo-4chloro-3-indolyl phosphate for $16 \mathrm{~h}$. Unbound complex was removed by washing, and sections were counterstained with eosin. ISH was performed on 15 colorectal cancers (six expressing HPP1 by RT-PCR, six in which no expression was seen, and three with no RT-PCR results), one adenoma, and six hyperplastic polyps as well as 18 normal colonic mucosa samples.

Immunohistochemistry of $\alpha$ Smooth Muscle Actin (SMA). Intestinal sections were incubated with a mouse monoclonal antibody to $\alpha$-SMA (1:400; Dako), followed by a biotinylated rabbit antimouse IgG as the secondary antibody, as previously described. The detection system used was a Dako streptavidin-biotin complex/horseradish peroxidase kit, with 3,3-diaminobenzidine tetrahydrochloride as the chromogenic substrate. Sections were counterstained with eosin (27).

Loss of Heterozygosity (LOH) Studies. Coding region primers (HPC and $H P Z$ ) were used to find the boundary between exons 1 and 2 by comparing the sequences obtained after amplification of both genomic and cDNA. Sequencing of this boundary in genomic DNA revealed a CT repeat in intron 1 (GenBank accession no. AF264150). The CT repeat was amplified with primers designed to produce a fragment of $244 \mathrm{bp}$ (HPJ 5'GCTGATTCCTGGCAAAGGTGCC-3' and HPP 5'-CTCAACTCCCCTGTACTACCTTGA- $3^{\prime}$ ) in 50 blood donor controls and in paired samples of normal and tumor DNA. Allele loss was analyzed in 55 colorectal cancers, 10 adenomatous polyps, and 27 hyperplastic polyps as described (17).

Chromosomal Localization of HPP1. The cDNA fragment amplified by the primers HPA and HPW was cloned into a pGEM-T vector (Promega) and used to find the chromosomal location of HPP1 by fluorescence ISH. The probe was nick-translated with biotin14-dATP and hybridized in situ at a final concentration of 20 $\mathrm{ng} / \mu \mathrm{l}$ to metaphases from two normal males. The fluorescence ISH method was modified from that previously described (29) in that chromosomes were stained before analysis with both propidium iodide and 4',6-diamidino-2-phenylindole. Images of metaphase preparations were captured by a cooled chargecoupled device camera using the chromoscan image collection and enhancement system (Applied Imaging, Newcastle, U.K.).

\section{Results}

Global Methylation Assay and Identification of HPP1. Using a screening assay on a small selected group of samples, several fragments were found to have differential methylation in both colorectal polyps and cancers. The most frequently methylated of these fragments is shown in Fig. $1 b$ and was methylated in both polyps and cancers but only rarely in normal mucosa. The methylated fragment was $370 \mathrm{bp}$ in length and contained a dense cluster of $49 \mathrm{CpG}$ sites, which spanned the 5' UTR and the first exon of a gene we have called HPP1, as it was initially isolated from a patient with hyperplastic polyposis (GenBank accession no. AF264150; Fig. 1c). The cluster qualified as a $\mathrm{CpG}$ island under standard criteria (30). Sequencing of overlapping cDNA fragments spanning a portion of the 5' UTR and $755 \mathrm{bp}$ of coding sequence, which included the methylated fragment, revealed that the fragment showed greater than $95 \%$ homology to six other cDNA sequences [TMP-2 (GenBank accession no. E14369), tomoregulin (Genbank accession no. AB004064), TMEFF2 (GenBank accession no. AB017269), DKFZp564L1878 (GenBank accession no. HSM802469), TPEF (GenBank accession nos. AF242222 and AF242221; ref. 31), and TENB2 (GenBank accession no. AF179274)]. Analysis of the translated sequences revealed follistatin domains and a trans- 
(a)
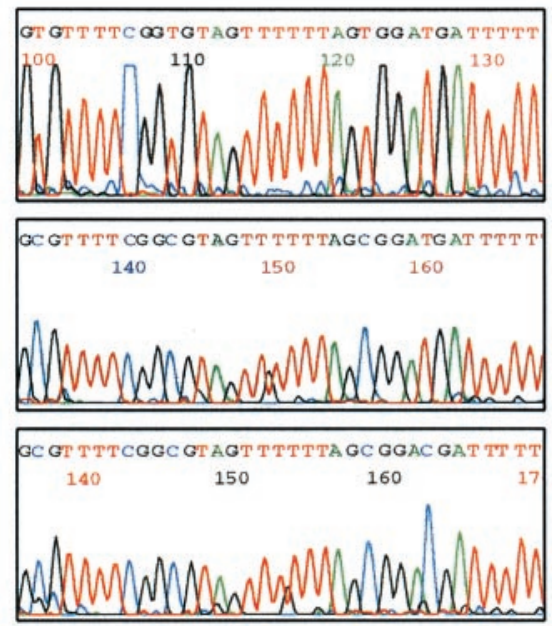

(c)
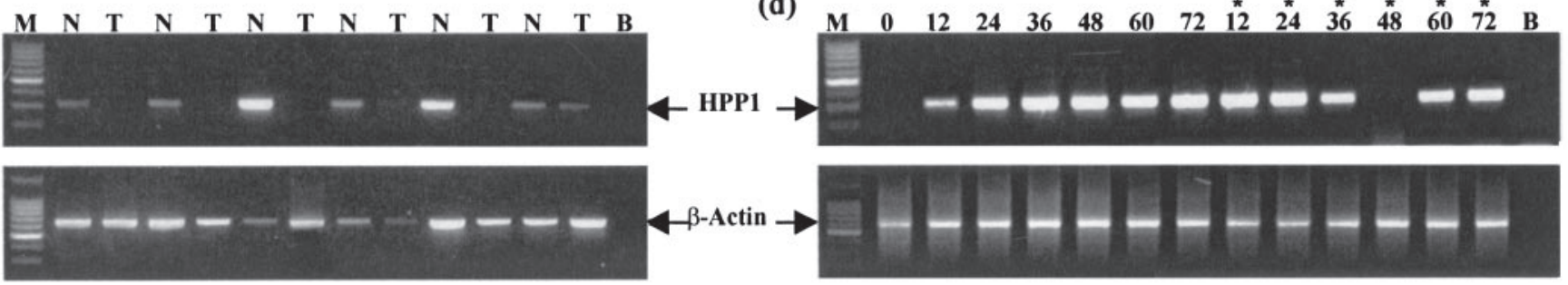

(d)

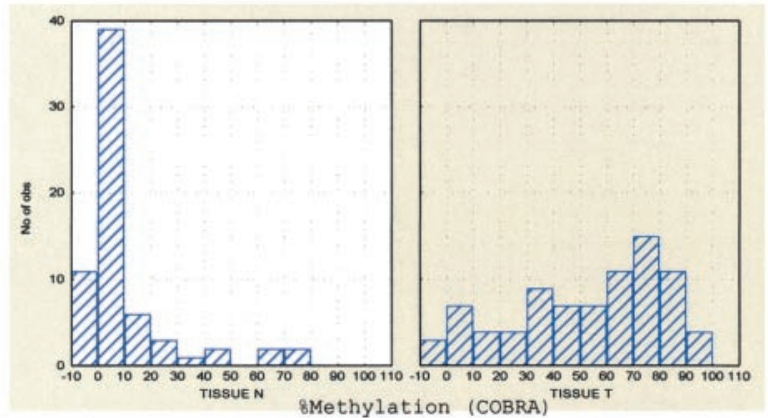

(b)
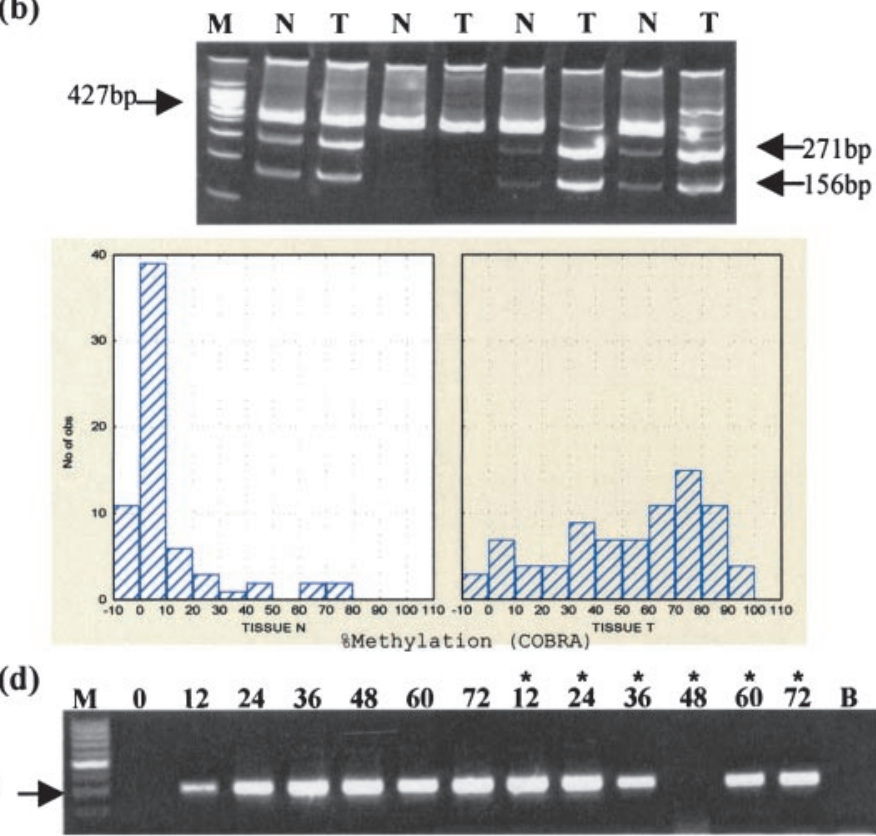

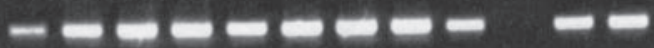

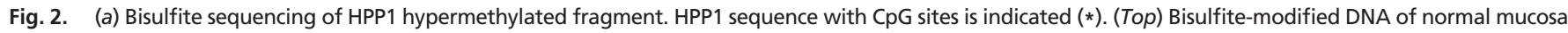

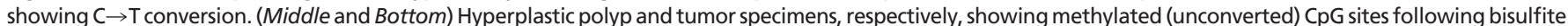

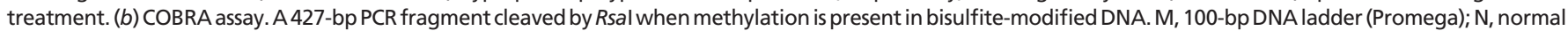

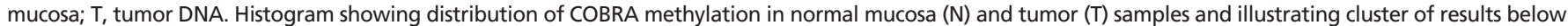

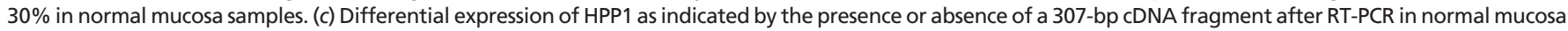

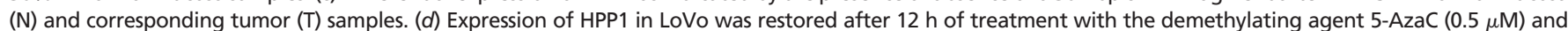

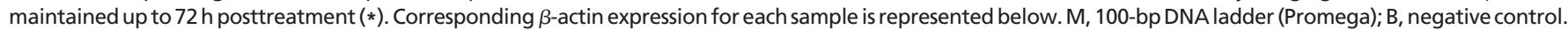

membrane region as described (GenBank accession no. AAD55776).

RACE. We used 3' RACE to identify the balance of the gene; this resulted in the isolation of two coding region variants of HPP1 (Fig. 1d). The most frequently isolated clone was HPP1- $A$, which would be predicted to produce a full-length transcript with $>95 \%$ homology to TENB2. The coding region of the less frequently isolated form $H P P 1-B$ was homologous to DKFZp564L1878, a clone containing a 57-bp insertion after nucleotide 1028 with three STOP signals (GenBank accession no. HSM802469; Fig. 1e). These signals would be predicted to truncate the protein just short of the cytoplasmic tail domain. Both variants were found in pooled normal mucosa, whereas in pooled HPP1 expressing cancer, only HPP1-A was observed. Variations in the $3^{\prime}$ UTR were seen in forms isolated from both normal and tumor. Clones homologous to the tomoregulin $3^{\prime}$-coding region were not isolated.

Confirmation of HPP1 as a Methylation Target. Bisulfite sequencing was performed in the 5' UTR of HPP1 in 2-5 clones each of a subset of neoplastic samples (six colorectal cancers, three hyperplastic polyps), which showed methylation by the global methylation assay, and five normal mucosal samples, which were negative by this assay (Fig. 2a). The fragment sequenced included $44 \mathrm{CpG}$ sites and was densely methylated. In those nine DNA samples in which methylation was detected by AP-PCR, an average of $96 \%$ of $\mathrm{CpG}$ sites was methylated per clone. All clones were methylated at the RsaI site used in the COBRA assay. The
COBRA assay was used to confirm HPP1-specific methylation (Fig. $2 b$ ). Methylation levels of $>30 \%$ were observed in 46 of 55 $(84 \%)$ colorectal cancers, six of nine $(66 \%)$ adenomas, and 17 of $27(63 \%)$ hyperplastic polyps. When cancers were analyzed with respect to MSI status, 17 of $19(89 \%)$ MSI-high, 13 of $18(72 \%)$ MSI-low, and 16 of $18(88 \%)$ microsatellite stable tumors showed $>30 \%$ methylation. Methylation occurred at low levels (less than $30 \%$ ) in 48 of $55(87 \%)$ normal mucosa samples. Seven normal samples showed methylation levels of $>30 \%$, and five of these occurred in patients with an MSI-high tumor $(P=0.041$, Fisher's Exact test; Fig. 2b). Analysis of COBRA values in normal mucosa as a continuous variable showed no significant correlation with age, sex, or site of sampling in the colon. In addition, there was no correlation between levels of HPP1 methylation in cancers and size, site, or MSI status of the tumor or age and sex of the patient, whether COBRA levels were analyzed as a continuous or discrete variable. Exposure to the demethylating agent 5-AzaC restored the expression of HPP1 in the two colorectal cancer cell lines. HPP1 expression was observed $12 \mathrm{~h}$ after initial treatment and was still present $72 \mathrm{~h}$ after the demethylating agent was removed (Fig. 2d).

RT-PCR and Correlation with COBRA. HPP1 was expressed in 28 of $30(93 \%)$ normal mucosa samples but in only seven of $30(23 \%)$ corresponding colorectal cancers tested (Fig. 2c). This represents a significant decrease in expression in cancers $(P=<0.001$, Fisher's Exact test). Cancers lost expression uniformly across MSI classes. A significant correlation was found between the level of methylation and the expression of HPP1 $(r=0.67, P<$ 


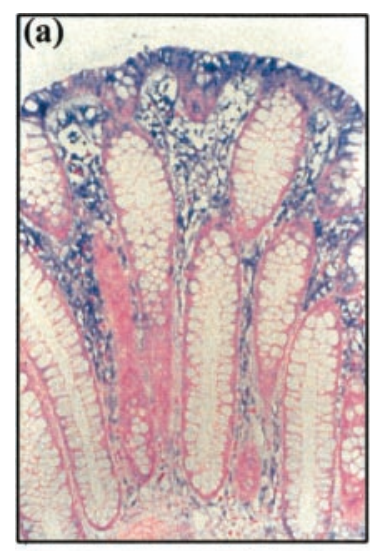

(b)
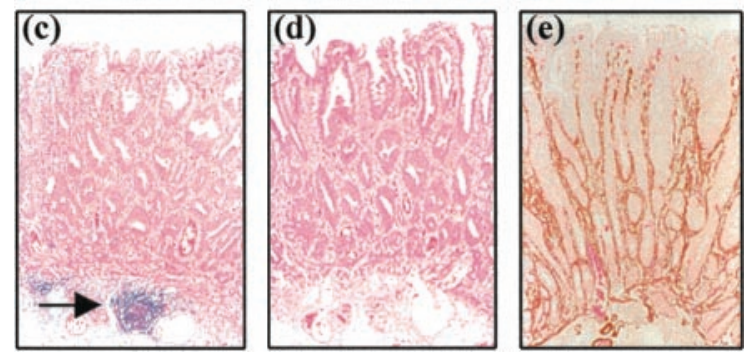

Fig. 3. ISH of HPP1 in normal mucosa ( $a$, antisense; $b$, sense riboprobes) and hyperplastic polyp ( $c$, antisense; $d$, sense riboprobes). Lymphoid aggregates, indicated by the arrow, act as an internal positive control. Pericryptal myofibroblasts express SMA (e) by immunohistochemistry despite losing expression of HPP1 in hyperplastic polyp.

0.001). Expression also was extinguished in one adenoma and one hyperplastic polyp on which tissue was available for RT-PCR analysis. Both lesions showed high methylation levels of 73 and $90 \%$, respectively.

ISH of HPP1 in Colorectal Tissue. Expression of HPP1 was observed in the pericryptal myofibroblasts and other stromal cells of normal colonic mucosa, and in epithelium where it was accentuated in terminally differentiated cells of the upper crypts. Expression also was seen in B-lymphocytes in gut-associated lymphoid tissue, scattered fibroblasts in the submucosa, ganglion cells, and endothelial cells (Fig. $3 a$ and $b$ ). Small hyperplastic polyps $(n=4)$ retained the patterns of expression seen in normal mucosa. In larger hyperplastic polyps $(n=2)$ and the single adenoma, HPP1 was not expressed in epithelia and stroma but was detected in lymphoid aggregates (Fig. $3 c$ and $d$ ). Junctions between normal mucosa and polyp showed a transition zone adjacent to the polyp in which expression gradually was extinguished. In cancers negative for HPP1 by RT-PCR $(n=6)$, there was only trace expression with the exception of one sample that showed moderate expression. In the cancers expressing HPP1 by both RT-PCR and ISH $(n=6)$, expression by ISH was more obvious in epithelium than stroma, although the stroma remained weakly positive.

Immunohistochemistry of $\alpha$ SMA. The pericryptal myofibroblasts are strap-like cells running close to and parallel with the crypt vertical axis. Unlike other stromal cells, these expressed HPP1 as well as SMA. Cells that expressed SMA but not HPP1 included smooth muscle cells in muscularis mucosae and vessel walls. Cancer and polyp myofibroblasts were also shown to express SMA, indicating that these cells were still represented in neoplastic lesions when HPP1 expression was abolished (Fig. 3e).

Chromosomal Localization of HPP1. Twenty metaphases from the first normal male were examined for fluorescent signal. All of these metaphases showed signal on one or both chromatids of chromo- (a)

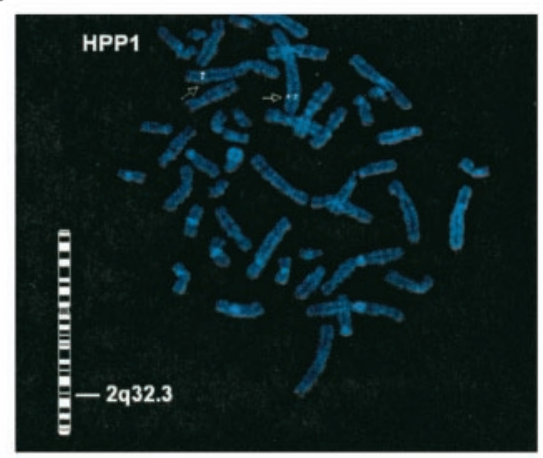

(b)

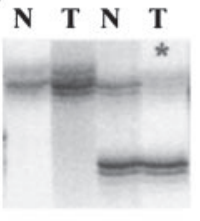

N $\quad$ P1 P2 P3

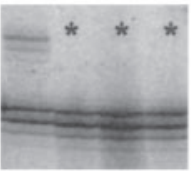

Fig. 4. (a) Metaphase showing fluorescence ISH with the HPP1 probe. Normal male chromosomes stained with 4',6-diamidino-2-phenylindole. Hybridization sites on chromosome 2 are indicated; $86 \%$ of the signal was at 2q32.3. (b) LOH analysis using the Microsatellite marker CT23i. Samples displaying LOH are indicated (*). N, normal mucosa; T, tumor; P, polyp DNA.

some 2 in the region 2q32-q33 (Fig. 4a). There was a total of 11 nonspecific background dots observed in the 20 metaphases. A similar result was obtained from hybridization HPP1 to 10 metaphases from the second normal male (data not shown).

LOH Studies. The first exon of HPP1 forms a boundary with exon 2 after $174 \mathrm{bp}$ of coding sequence. Within the intervening intron, there is an imperfect CT repeat of 23 units interrupted by a single AT after CT number 15 (GenBank accession no. AF264150). This microsatellite (CT23i) was found to be a 14-allele polymorphism with heterozygosity of 0.76 when tested on 50 unrelated Caucasian blood donors. LOH was observed in one of $22(5 \%)$ colorectal cancers, three of six $(50 \%)$ adenomas, and none of 22 hyperplastic polyps that were informative at CT23i (Fig. 4b). The single carcinoma in which $\mathrm{LOH}$ at HPP1 was observed was a sporadic, proximal, poorly differentiated signet ring cancer in a 67-yr-old female. The three adenomas that showed $\mathrm{LOH}$ at HPP1 all were derived from a 56-yr-old female with multiple adenomata. Both patients also demonstrated methylation of HPP1 in their neoplasms.

\section{Discussion}

Although the adenoma has long been recognized as the principal precancerous lesion in the colorectum, evidence is accumulating that the hyperplastic polyp may be the precursor of a subset of colorectal cancers. Neoplastic evolution has been well documented in hyperplastic polyposis $(10,11,13)$, and previous work from our laboratory has shown that hyperplastic polyps are linked to the microsatellite unstable pathway of carcinogenesis (16). Because MSI is associated with the hypermethylation phenotype (18), it is possible that the mechanism underlying the pathway of serrated neoplasia would be uncovered through the demonstration of differential methylation. Accordingly, we studied normal, hyperplastic, and malignant colorectal epithelium with a global methylation analysis technique and identified a gene HPP1.

We have demonstrated that the expression of HPP1 can be induced by the treatment of nonexpressing cell lines with a demethylating agent. A high degree of correlation was seen between the level of methylation and expression of HPP1 by RT-PCR; however, some discordant values were recorded. In five samples, high levels of methylation were accompanied by significant expression of HPP1. In these cases, it is possible that the expression may originate from stromal elements (see Fig. 3c). In a further five samples, there was no apparent methylation despite loss of expression, which may indicate the presence of an inactivating mutation leading to transcript instability, another form of transcriptional 
silencing, or an upstream mutational event. Alternatively, discordant values could be explained by atypical site-specific methylation or demethylation events at the single $\mathrm{CpG}$ dinucleotide probed in the COBRA assay (32). Allelic loss was not a common mechanism of inactivation of HPP1.

TMP-2, tomoregulin, TMEFF2 TPEF, and TENB2, which all show a high degree of homology to HPP1, are predicted to be transmembrane proteins containing follistatin, glycosaminoglycan binding, and epidermal growth factor-like domains. The expression of tomoregulin in adult tissues is apparently restricted to neurons and glial cells of the central nervous system and fibroblasts in the gastrointestinal mucosa (33), although we were unable to isolate tomoregulin from pooled normal mucosa by $3^{\prime}$ RACE. In view of its putative role as a ligand for erB-4 and its follistatin domains, it has been suggested that tomoregulin could be implicated in the coordination of proliferation, differentiation, or apoptosis of gastrointestinal tissues (33). Follistatin domains have been identified in other proteins such as osteonectin. Osteonectin (SPARC) is found in the lens of the eye and functions as an anti-adhesin disrupting cellular contact with matrix (34). When SPARC is rendered nonfunctional in the mouse, cataracts develop, and disordered maturation and migration of cells is observed (35). A molecule with homology to SPARC is predicted to underlie the normal development of gut mucosa. SPARC is known to interact with plateletderived growth factor (36), and platelet-derived growth factor Aand R-deficient mice show aberrant development of gut mucosa involving small intestinal villi and underlying mesenchyme (37). Transforming growth factor $\beta 3$, secreted by pericryptal myofibroblasts, is involved in the control of gut epithelial migration (38). The follistatin domains of SPARC have been implicated in its antiadhesive and antiproliferative properties, and it has been reported to induce the production of transforming growth factor $\beta 1$ (39). At least two pathways underlie intestinal mucosal growth and differentiation in which HPP1 could serve as an upstream regulator.

Methylation of HPP1 was widespread in the major types of colonic neoplasms and is unlikely to be the initiating lesion in all hyperplastic polyps because it is silenced only in larger lesions. Methylation of normal mucosa occurred more frequently in colons bearing high-level MSI cancers, a specific subtype that may evolve through hyperplastic lesions (10). Our demonstration of methyl-

1. Kinzler, K. W. \& Vogelstein, B. (1996) Cell 87, 159-170.

2. Herman, J. G., Umar, A., Polyak, K., Graff, J. R., Ahuja, N., Issa, J. P., Markowitz, S., Willson, J. K., Hamilton, S. R., Kinzler, K. W., et al. (1998) Proc. Natl. Acad. Sci. USA 95, 6870-6875. 3. Loukola, A., Salovaara, R., Kristo, P., Moisio, A. L., Kaariainen, H., Ahtola, H., Eskelinen, M., Harkonen, N., Julkunen, R., Kangas, E., et al. (1999) Am. J. Pathol. 155, 1849-1853.

4. Iino, H., Simms, L., Young, J., Arnold, J., Winship, I. M., Webb, S. I., Furlong, K. L., Leggett, B. \& Jass, J. R. (2000) Gut 47, 37-42.

5. Salahshor, S., Kressner, U., Pahlman, L., Glimelius, B., Lindmark, G. \& Lindblom, A. (1999) Genes Chromosomes Cancer 26, 247-252.

6. Olschwang, S., Hamelin, R., Laurentpuig, P., Thuille, B., Derycke, Y., Li, Y. J., Muzeau, F., Girodet, J., Salmon, R. J. \& Thomas, G. (1997) Proc. Natl. Acad. Sci. USA 94, 12122-12127.

7. Martinez, M. E., McPherson, R. S., Levin, B. \& Glober, G. A. (1997) Gastroenterology 113, 423-429.

8. Barkla, D. H. \& Gibson, P. R. (1999) Pathology 31, 230-238.

9. Hayashi, T. Yatani, R. Apostol, J. \& Stemmermann, G. N. (1974) Gastroenterology 66, 347-356.

10. Jass, J. R., Iino, H., Ruszkiewicz, A., Painter, D., Solomon, M. J., Koorey, D. J., Cohn, D., Furlong, K. L., Walsh, M. D., Palazzo, J., et al. (2000) Gut 47, 43-49.

11. Torlakovic, E. \& Snover, D. C. (1996) Gastroenterology 110, 748-755.

12. Otori, K., Oda, Y., Sugiyama, K., Hasebe, T., Mukai, K., Fujii, T., Tajiri, H., Yoshida, S. Fukushima, S. \& Esumi, H. (1997) Gut 40, 660-663.

13. Rashid, A., Houlihan, P. S., Booker, S., Petersen, G. S., Giardiello, F. M. \& Hamilton, S. R. (2000) Gastroenterology 119, 323-332.

14. Konishi, M., Kikuchiyanoshita, R., Tanaka, K., Muraoka, M., Onda, A., Okumura, Y., Kishi, N., Iwama, T., Mori, T., Koike, M., et al. (1996) Gastroenterology 111, 307-317.

15. Jen, J., Powell, S. M., Papadopoulos, N., Smith, K. J., Hamilton, S. R., Vogelstein, B. \& Kinzler, K. W. (1994) Cancer Res. 54, 5523-5526.

16. Iino, H., Jass, J. R., Simms, L. A., Young, J., Leggett, B., Ajioka, Y. \& Watanabe, H. (1999) J. Clin. Pathol. 52, 5-9.

17. Jass, J. R., Biden, K. G., Cummings, M. C., Simms, L. A., Walsh, M., Schoch, E., Meltzer, S. J., Wright, C., Searle, J., Young, J. \& Leggett, B. A. (1999) J. Clin. Pathol, 52, 455-460.

18. Ahuja, N., Mohan, A. L., Li, Q., Stolker, J. M., Herman, J. G., Hamilton, S. R., Baylin, S. B. \& Issa, J. P. (1997) Cancer Res. 57, 3370-3374.

19. Toyota, M., Ahuja, N., Ohe-Toyota, M., Herman, J. G., Baylin, S. B. \& Issa, J. P. (1999) Proc Natl. Acad. Sci. USA 96, 8681-8686.

20. Jass, J. R., Do, K. A., Simms, L. A., Iino, H., Wynter, C., Pillay, S. P., Searle, J., Radford-Smith, G., Young, J. \& Leggett, B. (1998) Gut 42, 673-679.

21. Miller, S. A., Dykes, D. D. \& Polesky, H. F. (1988) Nucleic Acids Res. 16, 1215. ation of HPP1 in hyperplastic polyps is of potential interest as little is known of the etiology of these common polyps. It is possible that silencing of HPP1 through promoter hypermethylation is a mechanism contributing to the development of at least a subset of hyperplastic polyps and that these may be the precursors of microsatellite unstable cancers. However, the finding of HPP1 methylation in many adenomas and cancers indicates a broader association in colorectal tumorigenesis. Cancers depend on complex interactions between the epithelial cells and their adjacent stroma and endothelium to support their growth (40). It is interesting that in neoplasms in which HPP1 expression was extinguished, this was observed in both colonic epithelium and stromal cells. Our results show that expression of HPP1 is silenced in the colon in both epithelium and fibroblasts in the course of tumorigenesis and that expression continues to be silenced in this manner in both chromosomal instability and MSI cancers.

In normal mucosa, HPP1 expression was particularly marked in pericryptal myofibroblasts, which are specialized cells displaying both myoid and fibroblastic features and acknowledged to be intimately associated with the coordinated maturation of crypt epithelium (41). They are the major mesenchymal elements of both adenomas and hyperplastic polyps and secrete growth factors influencing epithelial differentiation (42). Loss of expression of HPP1 in cancers could be explained by the known disappearance of pericryptal fibroblasts in the course of neoplastic progression (43). The continued presence of SMA in the pericrypt fibroblasts in hyperplastic polyps despite loss of HPP1 expression indicates that these cells are still present although altered.

In summary, demonstration of HPP1 methylation and loss of expression with RT-PCR in colorectal cancers is consistent with a role of hyperplastic polyps in the evolution of a subset of colorectal cancer $(10,16)$. The finding of methylation of HPP1 in most adenomas and cancers underlines a wider involvement of HPP1 in colorectal tumorigenesis. The structure and expression profile of HPP1 suggest that silencing by methylation may be implicated in an early disturbance of stromal-epithelial interaction.

We thank the National Health and Medical Research Council (Australia) and the Walter Paulsen Tumor Bank. This work was supported in part by National Institutes of Health Grant U01 CA-74778.

22. Liang, G., Salem, C. E., Yu, M. C., Nguyen, H. D Gonzales, F. A., Nguyen, T. T. Nichols, P. W. \& Jones, P. A. (1998) Genomics 53, 260-268.

23. Herman, J. G., Graff, J. R., Myohanen, S., Nelkin, B. D. \& Baylin, S. B. (1996) Proc. Natl. Acad. Sci. USA 93, 9821-9826.

24. Xiong, Z. \& Laird, P. W. (1997) Nucleic Acids Res. 25, 2532-2534.

25. Raff, T., van der Giet, M., Endemann, D., Wiederholt, T. \& Paul, M. (1997) BioTechniques 23, 456-460.

26. Karpf, A. R., Peterson, P. W., Rawlins, J. T., Dalley, B. K., Yang, O., Albertsen, H. \& Jones, D. A. (1999) Proc. Natl. Acad. Sci. USA 96, 14007-14012.

27. Ramm, G. A., Nair, V. G., Bridle, K. R., Shepherd, R. W. \& Crawford, D. H. (1998) Am. J. Pathol. 153, 527-535.

28. Rex, M. \& Scotting, P. J. (1994) Biochemica 3, 24-26.

29. Callen, D. F., Baker, E., Eyre, H. J., Chernos, J. E., Bell, J. A. \& Sutherland, G. R. (1990) Ann. Genet. 33, 219-221.

30. Gardiner-Garden, M. \& Frommer, M. (1987) J. Mol. Biol. 196, 261-282.

31. Liang, G., Robertson, K. D., Talmadge, C., Sumegi, J. \& Jones, P. A. (2000) Cancer Res. 60, 4907-4912.

32. Fitzpatrick, D. R., Shirley, K. M., McDonald, L. E., Bielefeldt-Ohmann, H., Kay, G. F. \& Kelso, A. (1998) J. Exp. Med. 188, 103-117.

33. Uchida, T., Wada, K., Akamatsu, T., Yonezawa, M., Noguchi, H., Mizoguchi, A., Kasuga, M. \& Sakamoto, C. (1999) Biochem. Biophys. Res. Commun. 266, 593-602.

34. Lane, T. F. \& Sage, E. H. (1994) FASEB J. 8, 163-173.

35. Bassuk, J. A., Birkebak, T., Rothmier, J. D., Clark, J. M., Bradshaw, A., Muchowski, P. J., Howe, C. C., Clark, J. I. \& Sage, E. H. (1999) Exp. Eye Res. 68, 321-331.

36. Raines, E. W., Lane, T. F., Iruela-Arispe, M. L., Ross, R. \& Sage, E. H. (1992) Proc. Natl. Acad. Sci. USA 89, 1281-1285.

37. Karlsson, L., Lindahl, P., Heath, J. K. \& Betsholtz, C. (2000) Development (Cambridge, U.K.) 127, 3457-3466.

38. McKaig, B. C., Makh, S. S., Hawkey, C. J., Podolsky, D. K. \& Mahida, Y. R. (1999) Am. J. Physiol. 276, G1087-G1093.

39. Bassuk, J. A., Pichler, R., Rothmier, J. D., Pippen, J., Gordon, K., Meek, R. L., Bradshaw, A. D., Lombardi, D., Strandjord, T. P., Reed, M., et al. (2000) Kidney Int. 57, 117-128.

40. Park, C. C., Bissell, M. J. \& Barcellos-Hoff, M. H. (2000) Mol. Med. Today 6, 324-329.

41. Podolsky, D. K. (1993) Am. J. Physiol. 264, G179-G186.

42. Powell, D. W., Mifflin, R. C., Valentich, J. D., Crowe, S. E., Saada, J. I. \& West, A. B. (1999) Am. J. Physiol. 277, C183-C201.

43. Li, A., Hasui, K., Yonezawa, S., Tanaka, S. \& Sato, E. (1999) Pathol. Int. 49, 426-434. 